\title{
Altered gut microbiota is associated with feeding intolerance in preterm infants
}

\author{
Xiaoyan $\mathrm{Hu}^{1,2}$, Yanmei Chang ${ }^{1 \oplus}$, Zijing Wang ${ }^{1 \oplus}$, Wenting $\mathrm{Bao}^{1 \oplus}$, Zailing $\mathrm{Li}^{1 \oplus}$ \\ ${ }^{1}$ Department of Pediatrics, Peking University Third Hospital, Beijing, China; ${ }^{2}$ Department of Pediatrics, Peking University Shenzhen \\ Hospital, Shenzhen, China.
}

\begin{abstract}
Background. Feeding intolerance (FI) is a common complication that may cause great harm to preterm infants. The mechanism of FI remains unclear, but probiotics may help prevent and alleviate its symptoms. We hypothesized that the alteration in gut microbiota may be associated with the development of FI. Our study aimed to investigate the association between gut microbiota and FI in preterm infants.

Methods. Ninety-seven preterm infants were divided into the FI group ( $\mathrm{N}=42)$ and the feeding tolerance (FT) group $(\mathrm{N}=55)$ depending on whether the infants were diagnosed with FI. The fecal samples of each infant were collected on the 7th day after birth. Fecal microbiota was analyzed by 165 rRNA sequencing. Plasma motilin were detected on day-1, 7, 14, and 21 .

Results. The microbial diversity of the FI group was significantly lower than that of the FT group. The abundance levels of phylum Proteobacteria, class Gammaproteobacteria, genera such as Escherichia/Shigella were higher in the FI group than in the FT group. The abundance levels of phylum Firmicutes, class Negativicutes, and genus Veillonella were higher in the FT group than in the FI group. The motilin levels on days 7 and 14 were negatively correlated with the FI-enriched genera Planomicrobium and Vibrio, respectively. Our study also found gut microbiota was correlated with FI clinical characteristics, including gestational age, birth weight, age of FI diagnosis, age of FI disappearance, and FI duration.

Conclusions. Altered gut microbiota is associated with FI in preterm infants. FI cases typically have lower microbial diversity, a decreased abundance of beneficial bacteria, and an increased abundance of pathogenic bacteria. Gut microbiota is correlated with the clinical characteristics of FI. The decrease in motilin secretion caused by some bacteria may lead to the occurrence of FI.
\end{abstract}

Key words: feeding intolerance, gut microbiota, 16S rRNA gene, preterm infant, motilin.

Feeding intolerance (FI) is a common gastrointestinal complication among preterm infants. The incidence of FI is about $60-70 \%$ in preterm infants with a birth weight (BW) of less than 2,000 g. ${ }^{1}$ FI in preterm infants is mainly manifested by gastric retention, vomiting, abdominal distension, and feeding plan disruption. It often leads to inadequate nutrient intake and postnatal growth retardation in preterm infants. ${ }^{2}$ Long-term

$\triangle$ Zailing Li

topbj163@sina.com

Received 18th February 2020, revised 22nd March 2020, 20th May 2020, accepted 28th July 2020. parenteral nutrition accompanied with FI also increases the incidence of complications such as nosocomial infection, metabolic disorders, and liver damage. ${ }^{3} \mathrm{FI}$ is an important cause leading to prolonged hospitalization and increased complications of preterm infants.

The human gut harbors a large number of microbes, which are named "gut microbiota." Normal gut microbiota plays an important role in human health. The gut microbiota of preterm infants differs dramatically from that of term infants, children, and adults. ${ }^{4}$ In preterm infants, gut microbiota disorders are associated with many diseases, such as necrotizing enterocolitis (NEC) and sepsis.5 FI may be an 
early manifestation of NEC. Supplementation with probiotics may be helpful in preventing FI in preterm infants, ${ }^{6}$ suggesting that gut microbiota plays a role in FI among preterm infants. However, this aspect has yet to be fully explored, and the relationship between gut microbiota and FI remains unclear. Our study aimed to investigate the relationship between gut microbiota and FI in preterm infants. It would help reveal new information related to the prevention and treatment of FI in preterm infants.

\section{Material and Methods}

\section{Diagnostic criteria of feeding intolerance}

Referring to the definition of FI by Moore et al., ${ }^{7}$ we defined infants who met two of the following conditions to have FI: (1) gastric residual volume (GRV) of more than $50 \%$ of the previous feeding volume ( $\geq$ twice within 24 h); (2) emesis, abdominal distention, or both; and (3) disruption of the patient's feeding plan presented as reduced or suspended feeding. We considered that FI disappeared when all of these conditions were no longer observed in 24 h. According to the 2015 Canadian Feeding Guideline, ${ }^{8}$ GRV was checked only after a minimum feed volume (per feed) was attained (minimum volume: $500-749 \mathrm{~g}: 3 \mathrm{ml}, 750-1000 \mathrm{~g}$ : $4 \mathrm{ml},>1000 \mathrm{~g}: 5 \mathrm{ml})$.

\section{Study design and participants}

This study is a single-center cross-sectional study. The inclusion criteria were as follows: (1) infants with gestational age (GA) of $\leq 34$ weeks; (2) infants who were born between June and December 2018; and (3) infants who were admitted to the Neonatal Intensive Care Unit (NICU) of Peking University Third Hospital within $2 \mathrm{~h}$ after birth. The exclusion criteria included the following: (1) infants who were not fed within $24 \mathrm{~h}$ after birth; (2) infants who died or gave up treatment within 7 days of birth; (3) infants who suffered from congenital gastrointestinal malformations or hereditary metabolic diseases; and (4) infants whose guardians did not sign an informed consent.

All preterm infants in our study were fed in accordance with the 2015 Canadian Feeding Guideline. ${ }^{8}$ Each infant was fed within $24 \mathrm{~h}$ after birth. Breast milk was the first choice for the infants; however, if their mothers could not produce breast milk, preterm formula was considered. In our study, no probiotics or erythromycin was given to the infants within 1 week. All the subjects who met the inclusion and exclusion criteria in our study were divided into the FI group and the feeding tolerance (FT) group in accordance with the diagnostic criteria of FI.

\section{Blood sample collection and motilin detection}

The residual blood samples of the infants were collected on day-1, 7, 14, and 21. A total of 178 blood samples were collected in our study. All blood samples were centrifuged and stored at $-80^{\circ} \mathrm{C}$ until they were assayed. Enzyme-linked immunosorbent assay (Multiskan FC, Thermo scientific, USA) was used for the quantitative measurement of motilin.

\section{Fecal sample collection and DNA extraction}

Fresh fecal samples were collected from diapers by using a sterile spoon and placed in sterile tubes on day 7 after birth. Then, the fecal samples were transported immediately to the laboratory on ice and stored at $-80^{\circ} \mathrm{C}$ prior to analysis.

DNA was extracted from each fecal sample by using a QIAamp fast DNA stool mini kit (Qiagen, Germany) in accordance with its improved protocol. In detail, $1 \mathrm{ml}$ of InhibitEX buffer and a proper amount of glass beads (0.5 $\mathrm{mm}$ diameter, Qiagen) were added to $200 \mathrm{mg}$ of each fecal sample. The mixture was homogenized and beaten at $60 \mathrm{~Hz}$ for 1 min. twice with a homogeneous instrument (FASTPREP-24, Aosheng Biotech, China). Afterward, DNA was purified in accordance with the manufacturer's instructions. 


\section{$16 S$ rRNA gene amplicon and sequencing}

The V3 to V4 region of bacterial $16 \mathrm{~S}$ ribosomal RNAgenes wereamplified through a polymerase chain reaction by using the barcoded primers 341F 5'-CCTACGGGRSGCAGCAG-3' and 806R 5'-GGACTACVVGGGTATCTAATC-3'. Amplicons were extracted from 2\% agarose gels and purified using an AxyPrep DNA gel extraction kit (Axygen Biosciences, Union City, CA, U.S.). All the quantified amplicons were pooled to equalize the concentrations for sequencing with Illumina MiSeq/HiSeq (Illumina, Inc., CA, USA). The 250 bp paired end reads were overlapped on their $3^{\prime}$ ends for concatenation into original longer tags by using PANDAseq (https://github.com/neufeld/ pandaseq, version 2.9).

\section{Ethical statement}

This study was approved by Peking University Third Hospital's Medical Science Research Ethics Committee (Ethical Approval No.: 05402), Beijing, China. The guardians of all the participants were informed of the purpose of the study, and they provided written informed consent in accordance with the Declaration of Helsinki.

\section{Statistical analysis}

SPSS (ver. 22.0, SPSS, Inc., Chicago, USA) and R (ver. 3.5.1, R Development Core Team, Vienna, Austria) were used for statistical analysis. Normally distributed quantitative data were represented as mean and standard deviation (SD), and Student's t-test for independent samples was used for comparison between groups. Qualitative data were represented as relative frequency and percent distribution, and chi-square test was used for comparison between groups. Microbiota data were summarized using $\alpha$-diversity and $\beta$-diversity measures to compare the microbial communities between groups. Shannon and Simpson index measurements were conducted to analyze $\alpha$-diversity and place weight on species richness and evenness. A Wilcoxon test ("wilcoxon. test" function in the $\mathrm{R}$ "stats" package) was used to determine significance. $\beta$-diversity indicated the shared diversity between bacterial populations in terms of ecological distance. Unweighted and weighted UniFrac distances were calculated for $\beta$-diversity analysis by using a table of operational taxonomic units (OTUs) and a phylogenetic tree. The unweighted UniFrac reflected the differences in community membership, whereas the weighted UniFrac mainly presented the differences in abundance. Ordination plots were generated through principal component analysis (PCOA). Differential abundance analysis was conducted at a genus level via a nonparametric Wilcoxon rank sum test, and adjusted for multiple testing by using the Benjamini-Hochberg procedure. ${ }^{9}$ Linear discriminant analysis (LDA) effect size (LEfSe) analysis (LEfSe v1.0) was carried out to determine the organisms most capable of explaining differences between the two groups. ${ }^{10}$ Different organisms with an LDA score cutoff of 2.0 were identified. Spearman correlation coefficients were calculated using $R$ (package "stats"), and a correlation heatmap was drawn with $\mathrm{R}$ (package "corrplot"). For result interpretation, $\mathrm{p}<0.05$ was considered statistically significant.

\section{Results}

A total of 97 subjects met the inclusion and exclusion criteria in our study. In accordance with the diagnostic criteria of FI, 55 cases were included in the FT group and 42 cases were placed in the FI group. The age of FI diagnosis was $2.9 \pm 0.9$ days ( $2-5$ days). The age of FI disappearance was $13.6 \pm 5.3$ days (8-26 days). The FI duration was $10.7 \pm 5.2$ days (4-24 days). There were no significant differences in gestational age, birth weight, gender, delivery mode, feeding type, and antibiotic exposure between the two groups ( $p>0.05$, Table I). Three infants in our study developed NEC (one in the FI group, two in the FT group) and one of them died of NEC. The details were shown in Table II. 
Table I. Characteristics of the study subjects.

\begin{tabular}{lccc}
\hline \multirow{2}{*}{ Characteristics } & \multicolumn{2}{c}{ Groups } & p-value \\
\cline { 2 - 3 } & Feeding intolerance $(\mathrm{N}=42)$ & Feeding tolerance $(\mathrm{N}=55)$ & \\
\hline Gestational age (week) & $30.0 \pm 2.1(25.3-34)$ & $30.6 \pm 1.9(25.2-34)$ & 0.132 \\
Birth weight (g) & $1270 \pm 343(700-2,070)$ & $1375 \pm 348(600-1,970)$ & 0.141 \\
Male, $\mathrm{n}(\%)$ & $22(52.4)$ & $37(63.7)$ & 0.137 \\
Caesarean section delivery, $\mathrm{n}(\%)$ & $26(61.9)$ & $41(74.5)$ & 0.181 \\
Human milk feeding mainly, $\mathrm{n}(\%)$ & $11(26.2)$ & $13(23.6)$ & 0.773 \\
Antibiotic exposure, $\mathrm{n}(\%)$ & $40(95.2)$ & $47(85.5)$ & 0.218 \\
Duration of antibiotic exposure (day) & $5.2 \pm 2.3(0-7)$ & $4.4 \pm 2.3(0-7)$ & 0.133 \\
\hline
\end{tabular}

Continuous variables are shown as means \pm standard deviation (minimum-maximum).

Human milk feeding mainly: human milk accounts for more than half of total milk intake.

Table II. Characteristics of the cases with necrotizing enterocolitis.

\begin{tabular}{lcccccccc}
\hline Case & Group & $\begin{array}{c}\text { GA } \\
\text { (week) }\end{array}$ & $\begin{array}{c}\text { BW } \\
\text { (g) }\end{array}$ & $\begin{array}{c}\text { Age at diagnosis } \\
\text { of FI (day) }\end{array}$ & $\begin{array}{c}\text { Age at disappearance } \\
\text { of FI (day) }\end{array}$ & $\begin{array}{c}\text { Age of diagnosis } \\
\text { of NEC (day) }\end{array}$ & $\begin{array}{c}\text { NEC } \\
\text { stage }\end{array}$ & $\begin{array}{c}\text { Outcome of } \\
\text { NEC }\end{array}$ \\
\hline 1 & FI & 28.6 & 1,100 & 2 & 15 & 26 & II & Cured \\
2 & FT & 29.6 & 1,400 & - & - & 11 & II & Cured \\
3 & FT & 28.1 & 910 & - & - & 12 & III & Died \\
\hline
\end{tabular}

BW: birth weight, FI: feeding intolerance, FT: feeding tolerance, GA: gestational age; NEC: necrotizing enterocolitis -: nonexistent.

\section{Microbial composition and abundance analysis}

The microbial composition of the two groups are shown in Fig. 1. At the phylum level, the top four phyla of the two groups were the same, and they were Proteobacteria, Firmicutes, Bacteroidetes, and Actinobacteria. In the FI group, the abundance percentages were as follows: $80.9 \%$ Proteobacteria, $11.5 \%$ Firmicutes, 5.1\% Bacteroidetes, and 1.9\% Actinobacteria. In the FT group, the abundance percentages were as follows: $69.5 \%$ Proteobacteria, $19.7 \%$ Firmicutes, $5.8 \%$ Bacteroidetes, and $4.0 \%$ Actinobacteria. At the genus level, the top four genera and their abundance percentages in the FI group were $59.7 \%$ Klebsiella, 8.2\% Serratia, 7.9\% Escherichia/Shigella, and 3.9\% Bacteroides. The top four genera and their abundance percentages in the FT group were 55.2\% Klebsiella, 9.5\% Serratia, 4.8\% Veillonella, and $4.1 \%$ Enterococcus. The relative abundance levels of Bifidobacterium in the FI and FT groups were $1.3 \%$ and $3.2 \%$, respectively. The abundance of Lactobacillus was very low and not among the top 20 genera found in both groups.

\section{Diversity comparison between the two groups}

Both $\alpha$ - and $\beta$-diversity indices were adopted to represent the comparison of microbiota diversity between the FI and FT groups. Simpson and Shannon indices were used to describe the $\alpha$-diversity of the gut microbiota between the two groups. The Simpson index of the FI group was significantly lower than that of the FT group ( $p=0.016$; Fig. 2A). This result indicated that the diversity of the gut microbiota in the FI group was significantly lower than that of the FT group. The Shannon index of the FI group was lower than that of the FT group, but their difference was not statistically significant $(p=0.053$; Fig. 2B). Significant difference in $\beta$-diversity between the two groups was detected by the weighted ( $p<0.05$, Fig. $2 \mathrm{C}$ ) but not the unweighted ( $p>0.05$, Fig. $2 \mathrm{D}$ ) PCOA (principal component analysis) based on the distance matrix of UniFrac. These results meant that the fecal microbial structure in the FI group was significantly different from that of the FT group because of the presence of OTUs. 
A

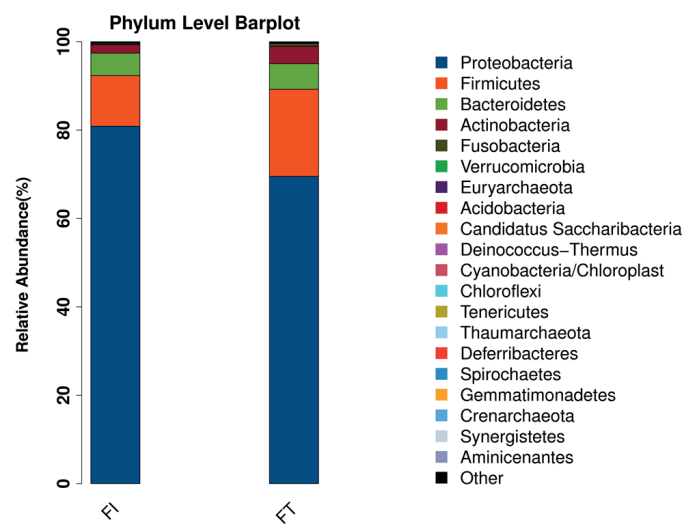

B

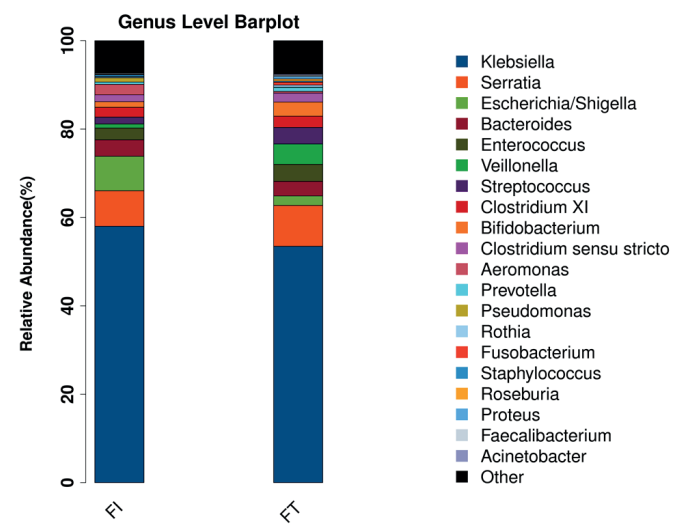

Fig. 1. Relative abundance of microbiota between the two groups at phylum and genus levels. The top 20 phyla (A) or genera (B) are displayed in different colors. The percentages of each taxon represent the relative abundance of the corresponding microbiota.

FI: feeding intolerance, FT: feeding tolerance.

A

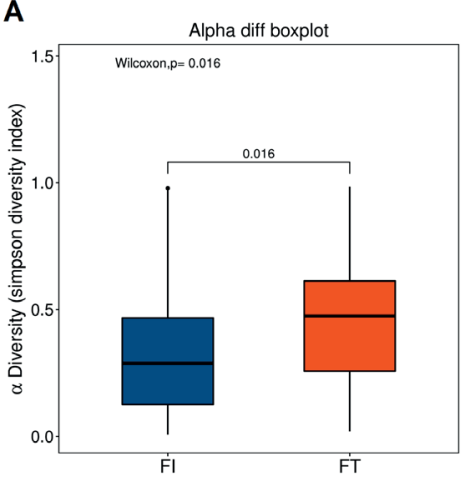

B

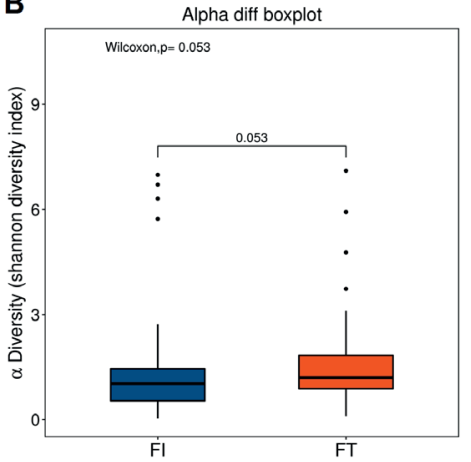

C

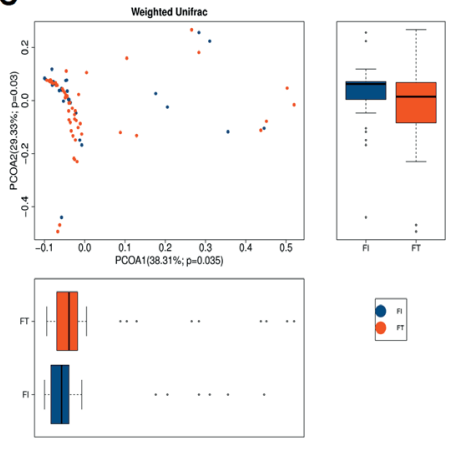

D

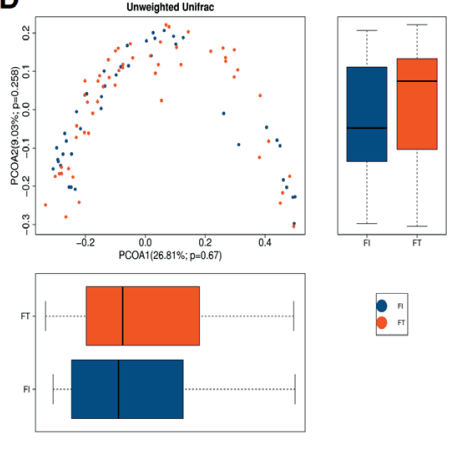

Fig. 2. Diversity of microbiota between the two groups. Box plots depict the differences in the fecal microbiome diversity indices between the FI (feeding intolerance) and FT (feeding tolerance) groups as indicated by the Simpson (A) and Shannon (B) indices based on OTU (operational taxonomic units) counts. Weighted (C) and unweighted (D) PCOA (principal component analysis) based on the distance matrix of the UniFrac dissimilarity of the fecal microbial communities in the FI and FT groups. Axes represent the two dimensions explaining the greatest proportion of variance in the communities. Each symbol represents a sample. The percentages in brackets respectively represent the contribution rate of each coordinate to the sample difference. Significant $\mathrm{p}$ values are indicated. 


\section{Differential microbiota between the two groups}

The microbiota with differential abundances between the FI and FT groups were analyzed in our study. LEfSe analysis was conducted to identify the differential microbiota by determining the presence and effect size of region-specific OTUs among different groups. A logarithmic LDA score cutoff of 2.0 was utilized to identify the important taxonomic differences between the FI and FT groups. Several taxa of the microbiota with differential abundances were detected between the FI and FT groups at different levels (Fig. 3). At the phylum level, the abundance of Firmicutes was higher in the FT group than in the FI group, and the abundance of Proteobacteria was higher in the FI group than in the FT group. At the class level, the abundance of Gammaproteobacteria in the FI group was higher than that in the FT group, whereas the abundance of Negativicutes in the FT group was higher than that in the FI group. At the genus level, the abundance of Veillonella was higher in the FT group than in the FI group, whereas the abundances of some genera, including Oceanisphaera, Janthinobacterium, Vibrio, Brachyspira, Oceanimonas, Petrobacter, Anaerovibrio, Planomicrobium, Cloacibacterium,
Vampirovibrio, Leptotrichia, Actinomycetospora, and Escherichia_Shigella, were higher in the FI group than in the FT group.

\section{Correlation between motilin and the microbiota}

Plasma motilin levels were detected on day-1, 7, 14, and 21. The correlation between motilin and the abovementioned differential bacteria was analyzed (Fig. 4). The results showed that the motilin level on day-7 was negatively correlated with Planomicrobium $(\mathrm{R}=-0.285, \mathrm{p}=0.038$, Fig. 4B), and the motilin level on day-14 was negatively correlated with Vibrio $(\mathrm{R}=-0.315, \mathrm{p}$ $=0.028$, Fig. 4C). Planomicrobium and Vibrio were the FI group-enriched genera. The motilin levels on days- 1 and 21 were not correlated with any of the differential bacteria.

\section{Correlation between the clinical characteristics of feeding intolerance and the microbiota}

A heatmap was used to demonstrate the correlation between the clinical characteristics of FI and the microbiota (all identified genera in the FI group). The heatmap showed that GA and BW were positively correlated with Escherichia/Shigella and Staphylococcus and

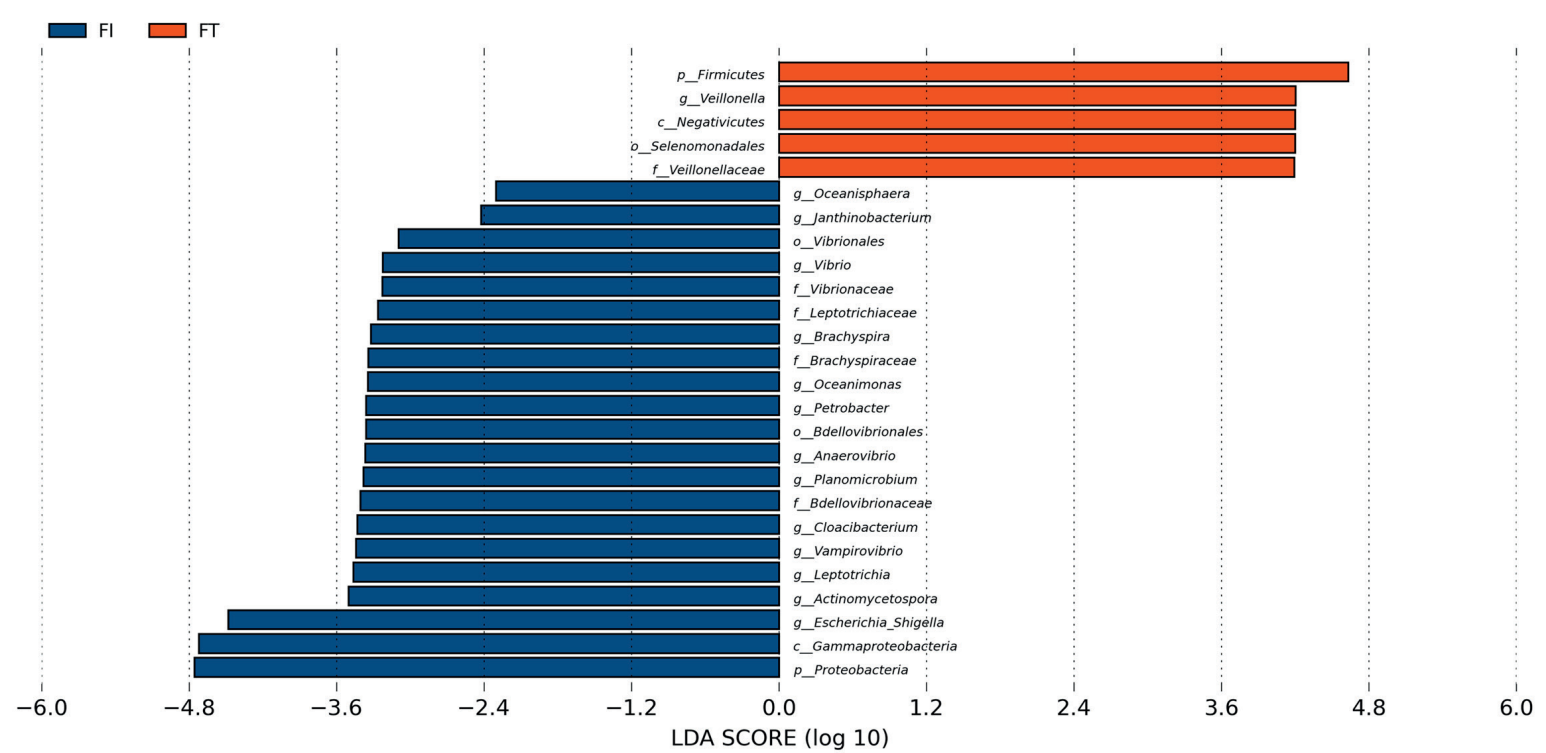

Fig. 3. Differential microbiota between the two groups. LEfSe analysis revealed significant bacterial differences in fecal microbiota between the FI (feeding intolerance) and FT (feeding tolerance) groups. LDA (linear discriminant analysis) scores $(\log 10)>2$ and $\mathrm{p}<0.05$ were listed. 
A

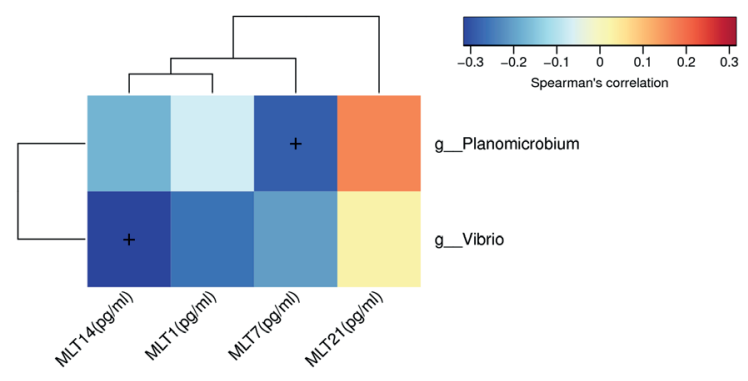

B

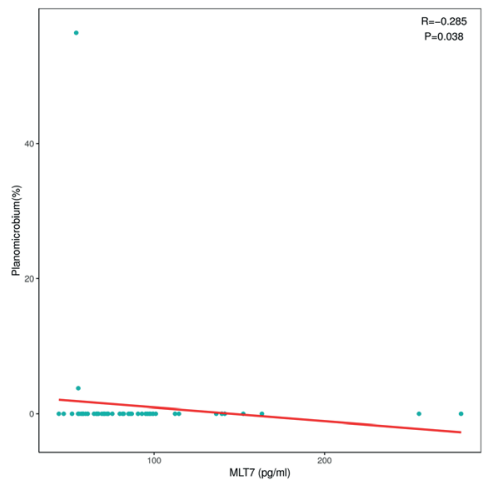

C

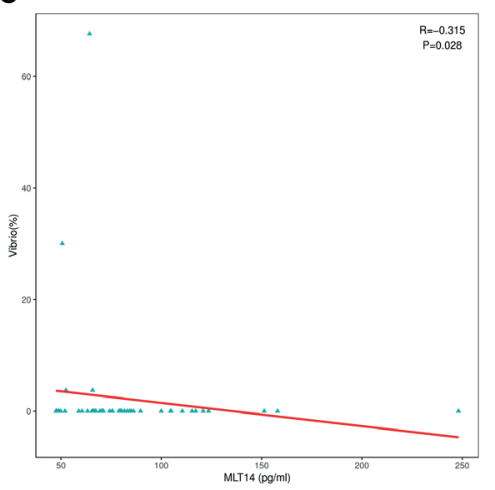

Fig. 4. Correlation between motilin and the microbiota. The correlation between motilin levels and the differential microbiota between the FI (feeding intolerance) and FT (feeding tolerance) groups was analyzed (A). The Spearman rank correlation $(\mathrm{R})$ and probability $(\mathrm{P})$ were used to evaluate statistical significance (B, C). The intensity of the color represents $r$ (correlation; negative score, blue; positive score, red).

Spearman test, ${ }^{+} \mathrm{p}<0.05$. MLT: motilin

negatively correlated with Klebsiella (Fig. 5). The age of FI diagnosis was positively correlated with Fructobacillus and Gluconacetobacter and negatively correlated with many genera such as Staphylococcus, Acinetobacter, and Proteus. The age of FI disappearance was negatively correlated with Enterococcus and positively correlated with several genera such as Leptotrichia and Gordonia. The FI duration was positively correlated with many genera such as Leptotrichia and Gordonia.

\section{Discussion}

Feeding intolerance has been a major problem for clinicians and infants' parents in recent years. The etiology and pathogenesis of FI in preterm infants are complicated and still unclear. The early colonization of the gut microbiota at birth can influence the correct ontogenesis of the gut barrier and motor and immune functions through a complex neuroendocrine cross-talk. ${ }^{11}$ Therefore, gut microbiota is associated with FI in preterm infants. However, this aspect has yet to be fully explored. Our study aimed to investigate the relationship between gut microbiota and FI in preterm infants. Because the clinical manifestation of FI is similar to early NEC, we need to distinguish FI from NEC. Three infants in our study did have NEC, and two were in the FT group. One infant in the FI group had NEC, but NEC occurred 11 days after FI disappeared. A large number of studies have shown that the dysbiosis of gut microbiota is closely related to the occurrence of NEC. However, there are few studies on the relationship between gut microbiota and feeding intolerance, which is exactly what our study focused on. 


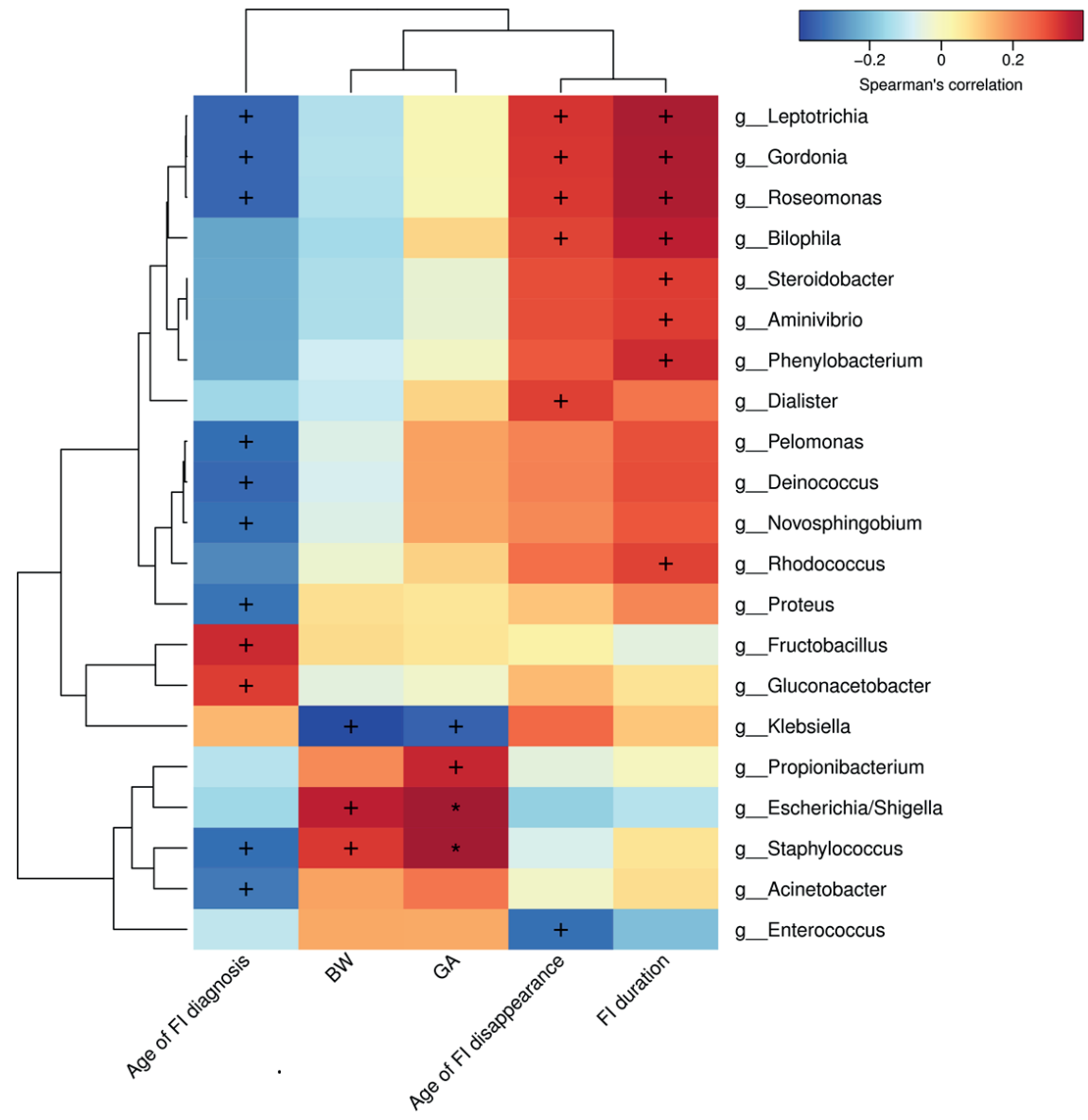

Fig. 5. Heatmap showing the correlation between the clinical characteristics of FI (feeding intolerance) and the microbiota. The heatmap shows the correlation between the clinical characteristics of FI and all the identified genera in the FI group. The intensity of the color represents r (correlation; negative score, blue; positive score, red).

Spearman test, ${ }^{+} \mathrm{p}<0.05,{ }^{*} \mathrm{p}<0.01$.

$\mathrm{BW}$ : birth weight, GA: gestational age.

The early colonization of the gut microbiota in preterm infants is influenced by many factors, and the most important ones are delivery mode, feeding type, and antibiotic exposure. ${ }^{12}$ Our study showed that there were no significant differences in GA, BW, delivery mode, feeding type and antibiotic exposure between the FI and FT groups. All of the subjects were hospitalized in one NICU and given similar treatment; hence, the gut microbiota of the two groups was comparable. The onset age of FI was $2-5$ days, and the curing age was 8-26 days; as such, collecting the stool samples on the 7th day was appropriate.
Our study showed that the top four phyla of both groups were Proteobacteria, Firmicutes, Bacteroidetes, and Actinobacteria (Fig. 1). These four phyla are the predominant gut microbiota in preterm infants. ${ }^{4,13}$ However, the relative abundances of the four phyla in the FI and FT groups were different: the relative abundance of Proteobacteria was higher in the FI group than in the FT group ( $80.9 \%$ vs. $69.5 \%)$, and the relative abundance of the Firmicutes was higher in the FT group than in the FI group $(19.7 \%$ vs. $11.5 \%)$. At the genus level, the top four genera in the FI group were Klebsiella, Serratia, Escherichial Shigella, and Bacteroides, and the top four 
genera in the FT group were Klebsiella, Serratia, Veillonella, and Enterococcus. These results showed that the composition and relative abundance of the gut microbiota were different between the FI and FT groups at different levels. Bifidobacterium and Lactobacillus are thought to be dominant in the gut of healthy infants after birth. ${ }^{14,15}$ However, in our study, the abundance of these bacteria was very low mainly because of factors such as preterm birth, cesarean delivery, antibiotic exposure, lack of breast milk feeding, and living in NICU with enriched pathogenic bacteria. ${ }^{16-19}$ Our results showed that the relative abundance of Bifidobacterium in the FI group was lower than that in the FT group $(1.3 \%$ vs. $3.2 \%$, respectively). This result might be related to the failure of gastrointestinal feeding in the FI group.

Microbial diversity plays an extremely important role in human health, that is, a low microbial diversity is associated with many diseases. In preterm infants, a low gut microbiota diversity is associated with late-onset sepsis. ${ }^{20}$ Our study compared the diversity of the FI and FT groups. The results showed that the Simpson index of the FI group was significantly lower than that of the FT group $(p=0.016)$. $\beta$-diversity analysis revealed that the weighted UniFracs in the FI and FT groups were significantly different $(p<0.05)$. This result indicated that the gut microbiota diversity in the FI group was significantly lower than that in the FT group. Our study also showed that the Shannon index of the FI group was lower than that of the FT group, but the difference was not statistically significant $(p=$ 0.053). This result might be related to our small sample size, which reduced the test efficiency. Microbial diversity significantly decreases when FI is present. ${ }^{21} \mathrm{Up}$ to now, studies on microbial diversity and FI are limited, but many studies have focused on the gut microbiota and NEC. FI may be an early manifestation of NEC. ${ }^{22}$ The characteristics of the gut microbiota of patients with FI may be similar to those of patients with NEC. Microbial diversity decreases significantly in NEC and further decreases as the severity of NEC increases. ${ }^{23-25}$ The results of our study also showed that gut microbiota diversity is closely related to FI in preterm infants.

The dysbiosis of the gut microbiota, often presented as an increase in pathogenic bacteria and a decrease in beneficial bacteria, is responsible for many diseases. Our study showed that the composition of the gut microbiota differed between the FI and FT groups at different levels. At the phylum level, the abundance of Proteobacteria in the FI group was higher than that in the FT group, and the abundance of Firmicutes in the FT group was higher than that in the FI group. A study of Yuan et al. ${ }^{21}$ showed that a low relative abundance of Firmicutes and a significantly increased relative abundance of Klebsiella (belonging to Proteobacteria) are detected in preterm infants when FI is diagnosed. This result was consistent with our study. Mai et al. ${ }^{26}$ reported that a $34 \%$ increase in the proportion of Proteobacteria and a 32\% decrease in Firmicutes are observed in infants 1 week before the diagnosis of NEC. The expansion of Proteobacteria is a microbial signature of gut dysbiosis and epithelial dysfunction. ${ }^{27}$ Besides the microbial difference at the phylum level, gut microbial differences at other levels were observed between the FI and FT groups. At the class level, the abundance of Gammaproteobacteria (belongs to phylum Proteobacteria) was higher in the FI group than in the FT group. By contrast, the abundance of Negativicutes (belonging to Firmicutes) was higher in the FT group than in the FI group. At the genus level, the abundance of Veillonella (belonging to Firmicutes) in the FT group was higher than that in the FI group. The abundance of several genera mainly belonging to Proteobacteria, including Escherichia_Shigella, in the FI group was higher than that in the FT group. Veillonella is considered a beneficial saccharolytic bacterial genus. And Escherichia Shigella is a common pathogenic bacterial genus related to many diseases, including gastroenteritis $^{28}$ and NEC. ${ }^{29}$ In general, the decreased abundance of beneficial bacteria and the increased abundance of pathogenic bacteria might be important factors leading to FI. 
Motilin is a very important hormone in gastrointestinal tract. A study has shown that preterm infants with FI have significantly lower motilin levels than those without FI. ${ }^{30}$ Gut microbiota may affect the secretion of gastrointestinal hormones. A randomized controlled study has demonstrated that preterm infants fed with prebiotic-enriched formula have significantly higher motilin levels, lesser gastric residues, and greater stool frequencies than those of infants fed with a common preterm formula. ${ }^{31}$ In our study, the motilin levels on day-7 and 14 were negatively correlated with FI-enriched Planomicrobium and Vibrio, respectively. However, the motilin levels on days 1 and 21 were not correlated with any of the differential bacteria between the FI and FT groups. The average age of FI diagnosis was 2.9 days, and the average age of FI disappearance was 13.6 days. This result meant that microbiota was correlated with motilin levels in the course of FI but not when FI disappeared or did not occur. Our results suggested that some microbiota, such as Planomicrobium and Vibrio, might reduce motilin secretion, leading to FI. This finding provided new information that could contribute to the treatment of FI in preterm infants and recommended a new direction for future research.

Some bacteria were correlated with FI clinical characteristics, including GA, BW, age of FI diagnosis, age of FI disappearance, and FI duration. GA and BW were positively correlated with Escherichia/Shigella and Staphylococcus and negatively correlated with Klebsiella. This result indicated that the composition of the gut microbiota changed with different GA and BW in preterm infants with FI. Some bacteria were also correlated with the age of FI diagnosis, age of FI disappearance, and FI duration. For instance, Leptotrichia, Gordonia, and Roseomonas were negatively correlated with the age of FI diagnosis, but they were positively correlated with the age of FI disappearance and FI duration. This finding indicated that these bacteria might lead to early onset, slow recovery, and long FI course. Inhibiting these bacteria might prevent and treat FI. Our study first revealed the correlation between microbiota and the clinical characteristics of FI in preterm infants. Further studies on these correlations will provide additional information about the prevention and treatment of FI in preterm infants.

Our study has some limitations. We only compared the gut microbiota of the FI and FT groups on the 7th day, and we did not analyze the microbiota before and after FI. Therefore, we could not understand the dynamic changes in the gut microbiota during the occurrence of FI. Our study could show that the gut microbiota was associated with FI in preterm infants, but we could not determine the causal relationship between them. Another limitation was that we conducted a single-centered research with a small sample size; therefore, the evidence level of the research results was limited. These limitations might be considered in future research.

In summary, our study revealed that the gut microbiota is associated with FI in preterm infants, and microbial diversity is low in FI cases. Our study also detected a decreased abundance of beneficial bacteria and an increased abundance of pathogenic bacteria in the FI group. However, the causal relationship between beneficial and pathogenic bacteria and the mechanism between gut microbiota changes and FI occurrence remain unknown. Future research should focus on these directions.

\section{Acknowledgement}

The authors would like to thank the nurses in the NICU of Peking University Third Hospital for their help in collecting fecal samples.

\section{REFERENCES}

1. Lodha A, Howlett A, Ahmed T, Moore AM. The role of interleukin- 6 and interleukin-8 circulating cytokines in differentiating between feeding intolerance and necrotizing enterocolitis in preterm infants. Am J Perinatol 2017; 34: 1286-1292. 
2. Morton DL, Hawthorne KM, Moore CE. Growth of infants with intestinal failure or feeding intolerance does not follow standard growth curves. J Nutr Metab 2017; 2017: 8052606.

3. Ng E, Shah VS. Erythromycin for the prevention and treatment of feeding intolerance in preterm infants. Cochrane Database Syst Rev 2008: CD001815.

4. Underwood MA, Sohn K. The microbiota of the extremely preterm infant. Clin Perinatol 2017; 44: 407-427.

5. Young GR, Smith DL, Embleton ND, et al. Reducing viability bias in analysis of gut microbiota in preterm infants at risk of NEC and sepsis. Front Cell Infect Microbiol 2017; 7: 237.

6. Indrio F, Riezzo G, Tafuri S, et al. Probiotic supplementation in preterm: feeding intolerance and hospital cost. Nutrients 2017; 9: 965.

7. Moore TA, Wilson ME. Feeding intolerance: a concept analysis. Adv Neonatal Care 2011; 11: 149154.

8. Dutta S, Singh B, Chessell L, et al. Guidelines for feeding very low birth weight infants. Nutrients 2015; 7: 423-442.

9. Benjamini Y, Hochberg Y. Controlling the false discovery rate: a practical and powerful approach to multiple testing. J R Stat Soc 1995; 57: 289-300.

10. Segata N, Izard J, Waldron L, et al. Metagenomic biomarker discovery and explanation. Genome Biol 2011; 12: R60.

11. Isolauri E. Development of healthy gut microbiota early in life. J Paediatr Child Health 2012; 48(Suppl 3): 1-6.

12. Saavedra JM, Dattilo AM. Early development of intestinal microbiota: implications for future health. Gastroenterol Clin North Am 2012; 41: 717-731.

13. Unger S, Stintzi A, Shah P, Mack D, O'Connor DL. Gut microbiota of the very-low-birth-weight infant. Pediatr Res 2015; 77: 205-213.

14. Harmsen HJ, Wildeboer-Veloo AC, Raangs GC, et al. Analysis of intestinal flora development in breastfed and formula-fed infants by using molecular identification and detection methods. J Pediatr Gastroenterol Nutr 2000; 30: 61-67.

15. Brooks B, Olm MR, Firek BA, et al. Strain-resolved analysis of hospital rooms and infants reveals overlap between the human and room microbiome. Nat Commun 2017; 8: 1814.

16. Penders J, Thijs C, Vink C, et al. Factors influencing the composition of the intestinal microbiota in early infancy. Pediatrics 2006; 118: 511-521.
17. Schumann A, Nutten S, Donnicola D, et al. Neonatal antibiotic treatment alters gastrointestinal tract developmental gene expression and intestinal barrier transcriptome. Physiol Genomics 2005; 23: 235-245.

18. Schwiertz A, Gruhl B, Löbnitz M, Michel P, Radke M, Blaut M. Development of the intestinal bacterial composition in hospitalized preterm infants in comparison with breast-fed, full-term infants. Pediatr Res 2003; 54: 393-399.

19. Huurre A, Kalliomaki M, Rautava S, Rinne M, Salminen S, Isolauri E. Mode of delivery - effects on gut microbiota and humoral immunity. Neonatology 2008; 93: 236-240.

20. Mai V, Torrazza RM, Ukhanova M, et al. Distortions in development of intestinal microbiota associated with late onset sepsis in preterm infants. PLoS One 2013; 8: e52876.

21. Yuan Z, Yan J, Wen H, Deng X, Li X, Su S. Feeding intolerance alters the gut microbiota of preterm infants. PLoS One 2019; 14: e0210609.

22. Lucchini R, Bizzarri B, Giampietro S, De Curtis M. Feeding intolerance in preterm infants. How to understand the warning signs. J Matern Fetal Neonatal Med 2011; 24(Suppl 1): 72-74.

23. Wang Y, Hoenig JD, Malin KJ, et al. 16S rRNA genebased analysis of fecal microbiota from preterm infants with and without necrotizing enterocolitis. ISME J 2009; 3: 944-954.

24. McMurtry VE, Gupta RW, Tran L, et al. Bacterial diversity and Clostridia abundance decrease with increasing severity of necrotizing enterocolitis. Microbiome 2015; 3: 11.

25. Hourigan SK, Ta A, Wong WS, et al. The microbiome in necrotizing enterocolitis: a case report in twins and minireview. Clin Ther 2016; 38: 747-753.

26. Mai V, Young CM, Ukhanova $M$, et al. Fecal microbiota in premature infants prior to necrotizing enterocolitis. PLoS One 2011; 6: e20647.

27. Litvak Y, Byndloss MX, Tsolis RM, Baumler AJ. Dysbiotic proteobacteria expansion: a microbial signature of epithelial dysfunction. Curr Opin Microbiol 2017; 39: 1-6.

28. Castano-Rodriguez N, Underwood AP, Merif J, et al. Gut microbiome analysis identifies potential etiological factors in acute gastroenteritis. Infect Immun 2018; 86: 100060-18.

29. Grishin A, Papillon S, Bell B, Wang J, Ford HR. The role of the intestinal microbiota in the pathogenesis of necrotizing enterocolitis. Semin Pediatr Surg 2013; 22: 69-75. 
30. Sun FJ, Huang RZ, Xu J, Liu GS. Relationship between plasma motilin level and feeding intolerance in preterm infants. Zhongguo Dang Dai Er Ke Za Zhi 2013; 15: 249-253.
31. Dasopoulou M, Briana DD, Boutsikou T, et al. Motilin and gastrin secretion and lipid profile in preterm neonates following prebiotics supplementation: a double-blind randomized controlled study. JPEN J Parenter Enteral Nutr 2015; 39: 359-368. 